\title{
Femtosecond-Laser-Assisted Cataract Surgery (FLACS)
}

\author{
Hui Sun, Andreas Fritz, Gerit Dröge, \\ Tobias Neuhann, and Josef F. Bille
}

\subsection{Introduction}

We obtain more than $80 \%$ of our information from the external world through vision. Good vision depends on the cornea and lens as refractive components. The crystalline lens is a transparent, biconvex structure in the eye that, along with the cornea, helps to refract light to be focused on the retina. Maintenance of lenticular shape and transparency is critical for refraction. The lens accounts for about one-third of the total refractive power of the eye. A slight change in the lenticular contour can result in refractive error. Small changes in the transparency or the shape of the lens can also cause visual distortion.

The lens is a part of the anterior segment of the eye. Anterior to the lens is the iris, which can control the amount of light that enters the eye.

\section{H. Sun}

Aerospace Information Research Institute, Chinese Academy of Sciences,

Beijing, China

\author{
A. Fritz · G. Dröge \\ University of Chinese Academy of Sciences, \\ Beijing, China \\ T. Neuhann \\ Augenklinik am Marienplatz, Munich, Germany \\ J. F. Bille $(\bowtie)$ \\ University of Heidelberg, Heidelberg, Germany
}

The lens is suspended in place by the zonules. Posterior to the lens is the vitreous body. The lens has an ellipsoid, biconvex shape. The anterior surface of the lens is less curved than its posterior surface. Normally, in adults the lens is $10 \mathrm{~mm}$ in diameter and has an axial length of about $4 \mathrm{~mm}$.

The lens has three main parts: the capsule, the epithelium, and the fibers. The lens capsule forms the outermost layer of the lens, and the lens fibers form the bulk of the interior. The lens epithelium is a simple cuboidal epithelium that is located in the anterior portion of the lens between the capsule and the fibers. The lens itself lacks nerves, blood vessels, or connective tissue. The refractive index of a human lens varies from about 1.406 in the center down to 1.386 in less dense part of the lens.

\subsection{Cataract and Surgery}

The most prevalent ocular disease and the major cause of blindness in the world is cataract. It is the third leading cause of preventable blindness in the Unites States. It is also the most frequently cited self-reported reason of visual impairment. Every year, there are more than eight million physician office visits due to visual disability from cataracts. Either clumps of protein or yellow-brown pigment may be deposited in the lens, reducing the transmission of light to the retina. Such opacity of the 
lens, whether it is a small local opacity or a diffuse general loss of transparency, is called a cataract. It must cause a significant reduction in visual acuity or a functional impairment to be clinically significant. Signs and symptoms of cataracts may include faded colors, blurry vision, and halos around lights, trouble with bright lights, and trouble seeing at night. Cataracts are most commonly caused by aging but may also occur due to diabetes mellitus, drugs, ultraviolet (UV) radiation, smoking, alcohol and nutrition. The three common types of this disease are nuclear, cortical, and posterior sub-capsular cataract. Visual inspection and assignment of numerical values to indicate severity is used to grade cataracts. A slit lamp is the regular tool for such an examination. The Oxford Clinical Cataract Classification and Grading System, the Johns Hopkins system, and the Lens Opacity Classification System (LOCS, LOCS II, and LOCS III) are alternative grading systems advocated for use in epidemiological studies of cataract [1-3]. Most people over the age of 60 have some degree of cataract formation. The extent of people's visual disability determines the treatment decision. If the cataract is not serious, surgery may not be needed. But, usually, there is no alternative to cataract surgery to correct visual impairment. In most cases, the standard of care in treating cataract is removal of the cataract by two types of surgical procedures: phacoemulsification (PE or phaco) and extra capsular cataract extraction. Following surgical removal of the crystalline lens, an artificial intraocular lens (IOL) is implanted. Cataract surgery is normally performed by an ophthalmologist with a fast procedure that causes little or no discomfort to the patient.

$\mathrm{PE}$ or phaco is the most common technique used in the United States today. It uses a machine with an ultrasonic probe to remove cataract. After the opening incision and anterior capsulotomy, an ultrasonic probe emulsifies the hard nucleus, enabling the ophthalmologist to remove the lens material using a suction device. The physical depth of the anterior chamber is maintained during this procedure. The opening is then enlarged to allow insertion of a posterior chamber IOL into the capsular bag. An IOL is usually implanted into the eye either through a small incision using a foldable IOL or through an enlarged incision using a polymethylmethacrylate (PMMA) lens.

After cataract surgery, the patient will be instructed to use anti-inflammatory eye drops for a few weeks. The eye will be mostly recovered within a week, and complete recovery takes about a month. This surgery has a high success rate and is the most common ophthalmic surgery procedure, with $\sim 19.5$ million procedures performed worldwide in 2011.

\subsection{History of Femtosecond- Laser-Assisted Cataract Surgery}

The application of ultrashort laser pulses to invivo ablation of cataractous lens tissue was first proposed in 1992 (US patent 5,246,435, Sept. 21, 1993 (J.F.Bille, D.Schanzlin): "Method for Removing Cataractous Material") and a related FDA-regulated initial clinical study was performed at the eye clinic of the University of Saint Louis (Fig. 14.1). The abstract of US 5,246,435 reads:

\footnotetext{
"A method for using an ophthalmic laser system to remove cataractous tissue from the lens capsule of an eye requires phacofragmentation of the lens tissue and subsequent aspiration of the treated tissue. More specifically, a cutting laser is used to create various strata of incisions through the lens tissue. Within each stratum, each incision is made in the direction from a posterior to an anterior position. The strata are stacked on each other in the posterior-anterior direction, and each includes a plurality of minute incisions. The most posterior stratum of incisions is created first by referencing the cutting laser back into the lens tissue from the posterior capsule. Subsequent, more anterior strata are created by referencing the cutting layer from the tissue treated by the previous stratum of incisions. In each stratum, the vapors which result from the incisions are allowed to infiltrate between the layers of the lens tissue to fragment and liquify the tissue. The liquified lens tissue is then aspirated."
} 

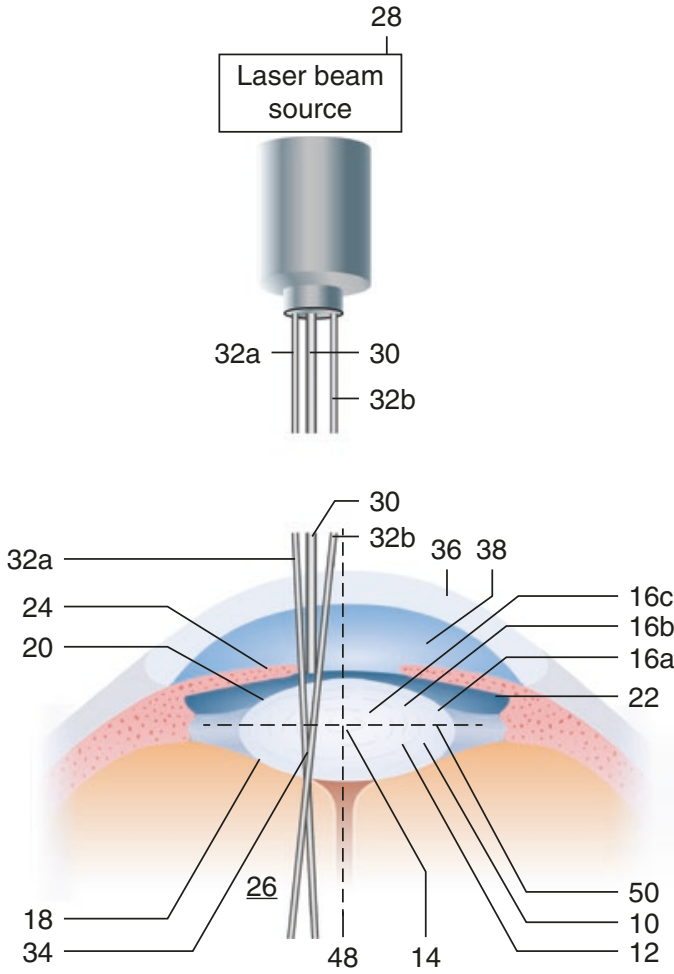

Fig. 14.1 Method for removing cataractous material, U.S. patent no. 5,246,435 [4]

Shortly before the expiration date of US patent 5,246,435, the commercialization of Femtosecond-Laser-Assisted cataract surgery was initiated by several ophthalmic laser companies.

\subsection{All-Solid-State Chirped- Pulse-Amplification Femtosecond Laser}

A femtosecond is the SI unit of time equal to $10^{-15}$ of a second. A femtosecond laser refers to a laser with pulse duration in the femtosecond range. Different types of lasers can produce femtosecond pulse, such as dye lasers, solid-state lasers, and fiber lasers. A solid-state laser is a laser that uses a gain medium that is a solid, and not a liquid, as in case of dye lasers, and not a gas, as in gas laser. Semiconductor-based lasers are also in the solid state but are generally con- sidered as a separate class of solid-state lasers. There are many hundreds of solid-state media in which laser action has been achieved, but relatively few types are widely used. Of these, the most common types are probably neodymiumdoped glass (Nd:glass) and neodymium-doped yttrium aluminum garnet (Nd:YAG). Typically, solid-state lasers are optically pumped, using either a flash lamp or laser diodes. Diodepumped solid-state lasers tend to be much more efficient, and have become much more common as the cost of high-power semiconductor lasers has decreased. A femtosecond laser has the following basic elements: a broadband gain medium, a laser cavity, an output coupler, a dispersive element, a phase modulator, and a gain/ loss process controlled by the pulse intensity or energy. The listed components are crucial for the function of the system. For example, the gain rod in an Nd:glass laser can cumulate the functions of gain, phase modulation, loss modulation, and gain modulation. The generation of femtosecond pulses often involves a dispersive mechanism of pulse compression; phase modulation broadens the pulse bandwidth, and dispersion eliminates the chirp and compresses the pulse.

A single femtosecond laser pulse is employed to ablate eye tissue to achieve enhanced precision and minimize collateral tissue effects during regular femtosecond-laser eye surgery. The threshold for a pulse-duration range of a few hundred femtoseconds is about $1-2 \mathrm{~J} / \mathrm{cm}^{2}$ [5]. The singlelaser pulse energy from the oscillator cannot satisfy this threshold requirement even though the laser beam can be focused very well. A technique called chirped pulse amplification (CPA) is used to amplify a femtosecond laser pulse and satisfy the intensity requirement for laser in situ keratomileusis (LASIK) surgery (Fig. 14.2). CPA is the current state-of-the-art technique that almost all of the highest-power lasers in the world currently utilize. It was originally introduced as a technique to increase the available power in radars in 1960s [6]. Because the nonlinear processes, such as self-focusing, can cause serious damage to the optic components if a femtosecond 

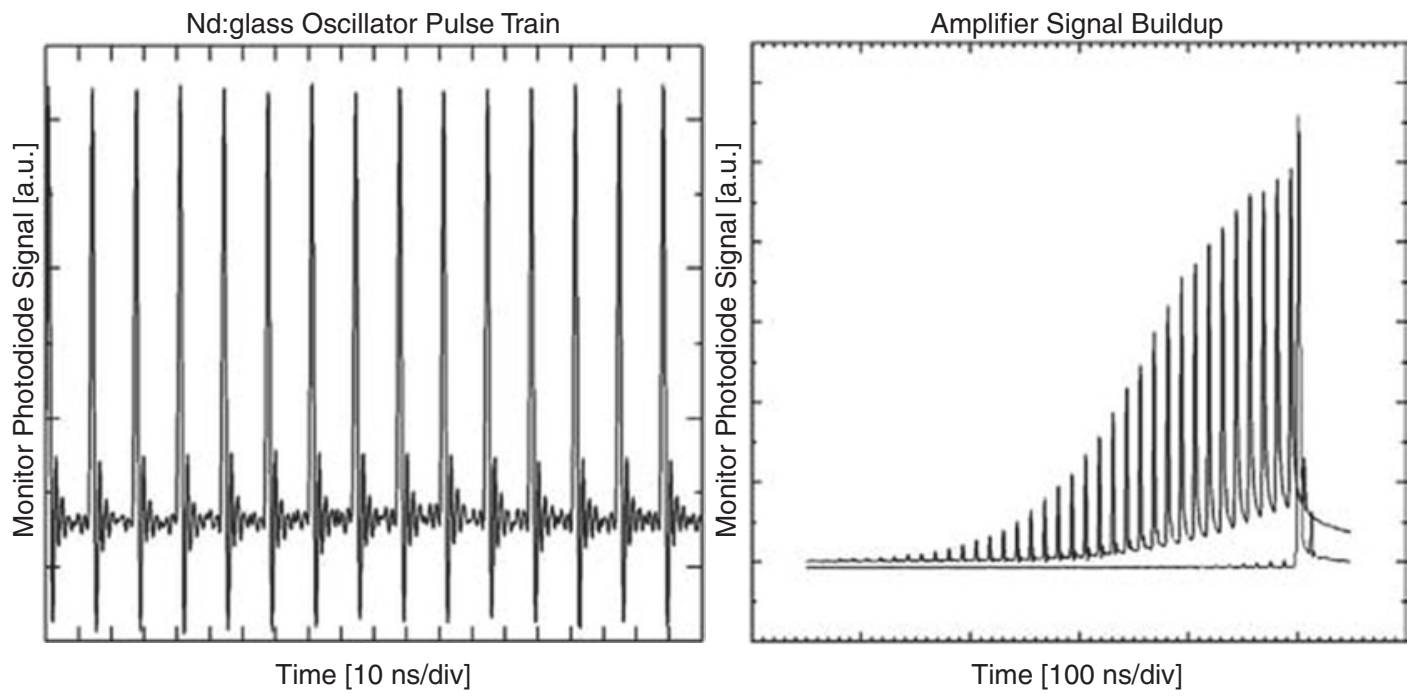

Fig. 14.2 Left: High repetition rate femtosecond pulse train. Right: Energy buildup during amplification and output signal

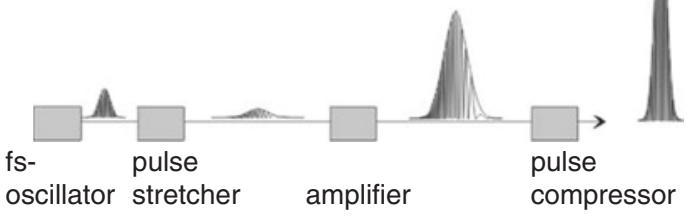

Fig. 14.3 Chirped pulse amplification (CPA) technique

laser pulse is directly amplified, the peak power of femtosecond laser pulses is limited before CPA can be achieved. CPA for femtosecond lasers was invented by Strickland and Mourou in the 1980s [7]. When employing the chirped pulse amplification technique, typically an increase of the pulse energy from the $\mathrm{nJ}$ to the $\mathrm{mJ}$ level at repetition rates of the order of $\mathrm{kHz}$ can be achieved, i.e., average powers of $\sim \mathrm{W}$ are obtained. A scheme of the chirped pulse amplification technique is shown in Fig. 14.3.

In order to keep the intensity of a femtosecond laser pulse below the threshold of the nonlinear effects inside the amplifier, the femtosecond laser pulse prior to introducing it to the amplifier is stretched out in time using a pair of gratings that are arranged so that the low-frequency component of the laser pulse travels a shorter path than the high frequency component does. This technique is called group velocity dispersion (GVD). After going through the grating pair, the laser pulse becomes positively chirped. The high-frequency component lags behind the low-frequency component and has longer pulse duration than the original one by a factor of $10^{3}-10^{5}$. Then the stretched pulse, whose intensity is sufficiently low, is safely introduced to the amplifier and is amplified by a factor of $10^{3}$ or more [8]. Finally, the amplified laser pulse is recompressed back to the femtosecond range through the reversal process of stretching, achieving a peak power orders of magnitude higher than those the laser systems could generate before the invention of the CPA technique. The physics concept behind the stretcher is GVD, which causes a short pulse of light to spread in time as a result of different frequency components of the pulse traveling at different velocities. There are three regular ways to achieve GVD for a femtosecond laser pulse: prisms, gratings, and a Gires-Tournois interferometer. However, a typical femtosecond laser CPA system requires that the pulse be stretched to several hundred picoseconds, which 
means that the different wavelength components must experience about $10 \mathrm{~cm}$ differences in path length. The most practical way to achieve this arrangement is with the grating-based stretcher and compressor.

\subsection{Femtosecond Laser Application Systems for Clinical Use}

To accurately control the power of ultrashort laser pulses for applications in ophthalmology, the laser system needs to be coupled with a precise, fast deflecting, and focusing unit as well as a high-contrast microscope suiting the needs of an ophthalmic surgeon.

After exiting the laser, the beam is coupled into the application arm. A mechanical shutter blocks the laser and opens only during the laser procedure. Each laser procedure is in principle defined by a three-dimensional data array of volume-elements (voxels) that will be ablated and a corresponding timeline that defines the ablation sequence. Consequently, the laser focus has to be precisely positioned in all three dimensions. For that purpose, a fully computercontrolled mirror scanning unit is employed (see Fig. 14.4). Optimized scan patterns are generated from a simple set of user-defined parameters (e.g., flap thickness and diameter, hinge angle in
pre-LASIK cutting of a flap) and performed by real-time control hardware. Behind the scanner unit, the beam passes an expanding telescope, increasing the laser beam diameter to achieve a tight focus behind the cutting lens. As the laser fluence has to be above the respective threshold for plasma-mediated ablation, the laser beam needs to be focused to a very small spot size of several micrometers to achieve an exact ablation. According to physical laws of optical lenses, the focus spot size of a beam decreases with larger entrance aperture of the focusing lens. The lateral ablation zone of the demonstrated scanning unit has a diameter of up to $10 \mathrm{~mm}$ in the cornea, with a focus shift range in $\mathrm{z}$ direction of up to $3 \mathrm{~mm}$. A schematic of the complete application system setup is shown in Fig. 14.4.

A surgical microscope, which is adapted to the system, provides the surgeon with a binocular, stereoscopic image to follow the process of the procedure. To support the handling needs in various surgical procedures, different field-ofview settings are provided by the microscope. In addition, a CCD camera is integrated into the microscope for monitoring and recording of the laser procedures. In case of FLACS systems, a computer-guided laser linked to an optical imaging system (e.g. OCT) performs the corneal incision, capsulotomy, and lens fragmentation steps, as well as the setup and the in-vivo control of the surgical procedure. In the next para-
Fig. 14.4 Application unit for performing ophthalmic surgery with femtosecond laser pulses

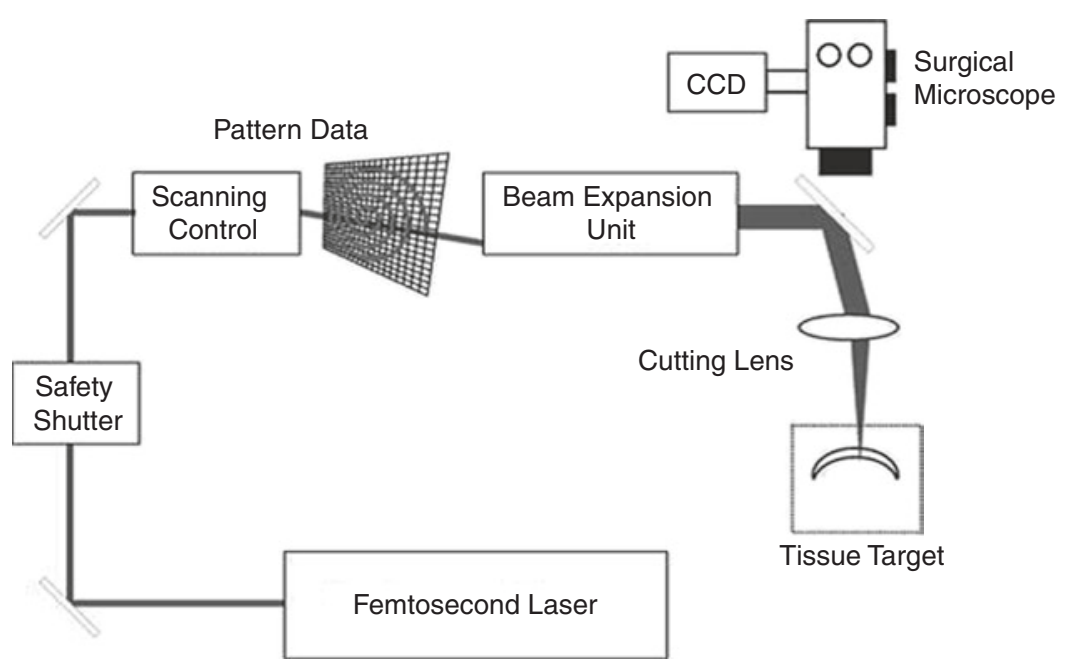


graph, the latest swept-source OCT technology which was developed by Heidelberg Engineering $\mathrm{GmbH}$, Heidelberg, Germany, and is applied in the VICTUS Femtosecond Laser Platform, is described.

Femtosecond laser systems have successfully entered the cataract surgery market since LenSx (Alcon Laboratories Inc.) introduced its first commercial system in 2008 as a promising new technological advance, which plays an everincreasing role in cataract surgery, automating the three main surgical steps: corneal incision, capsulotomy, and lens fragmentation. It is an innovative, growing new technology for cataract surgery due to the enhanced precision and minimized collateral tissue effects from femtosecond laser ablation. This attribute of femtosecond lasers is especially important for femtosecond laser cataract surgery, wherein the preservation of ocular structures such as the capsular bag is critical for good visual outcomes. The preliminary reports on the intraocular use of femtosecond lasers were promising [9-11]. The first clinical report of a human eye treated by femtosecond laser cataract surgery was in Hungary in 2008 [12]. There has been increasing interest in the use of femtosecond lasers in cataract surgery after this first report. The FDA approved the usage of femtosecond lasers for cataract surgery in 2010. The application of femtosecond lasers in cataract surgery increased dramatically after the LenSx system was cleared for use by the FDA. In only a few years, femtosecond lasers have become relevant in cataract surgery in clinics as an opportunity to improve the quality of the surgical procedure. In a relatively short period of time, the LenSx femtosecond laser system has been used in more than 200,000 procedures worldwide to date [13]. Multiple commercial femtosecond lasers have been cleared for use by the U.S. FDA for cataract surgery, including use in creating corneal incision, capsulotomy and lens fragmentation. These include LenSx (Alcon Laboratories Inc.), Catalys (Abbott Medical Optics), LensAR (LensAR Inc., Orlando, Florida), Victus (Technolas Perfect Vision and Bausch \& Lomb, Rochester, New York) and Femto LDV (Ziemer Ophthalmic
Systems AG). They are solid-state femtosecond lasers integrated within an imaging subsystem. The main principles are the same, but they differ in versatility, docking, speed of action, etc.

\subsection{Optical Coherence Tomography in Ophthalmic Applications}

Optical coherence tomography (OCT) is an interferometric imaging technology that was introduced by David Huang et al. in 1991 at the MIT [14]. It is frequently referred to as ultrasound with light. It enables a much higher resolution $(\mu \mathrm{m})$ than ultrasound while still allowing for imaging depths of several millimeters.

In order to obtain the image, the OCT light is split into two arms, the sample arm aiming at the item of interest (e.g. the patient eye), and the reference arm, e.g. a mirror. The reflected light of both arms is then brought together to form an interference pattern. Processing this pattern leads to a depth reflectivity profile, the so called A-scan. By scanning the beam across the sample, multiple A-scans can be obtained and combined into a cross section, the so called B-scan.

The first implementation of OCT was the so called time-domain OCT, in which a low coherence light source is used (Fig. 14.5a). Therefore interference only occurs as long as the path lengths of sample and reference arm are matched better than the coherence length of the light source. This coherence gating allows depth discrimination by reference arm length tuning, typically realized by a motorized mirror stage. This mechanical movement limits the A-scan rate to the lower $\mathrm{KHz}$ range.

In contrast to this, in Fourier-domain OCT the whole broadband interference pattern is acquired spectrally encoded. This is achieved by either a broadband light source and dispersive detector (spatially encoded Fourier-domain OCT, often called spectral-domain OCT) (Fig. 14.5b), or by tunable narrowband light source and a point detector (time encoded Fourier domain OCT, often called swept-source OCT) (Fig. 14.5c). Based on the Wiener-Khinchin theorem the 

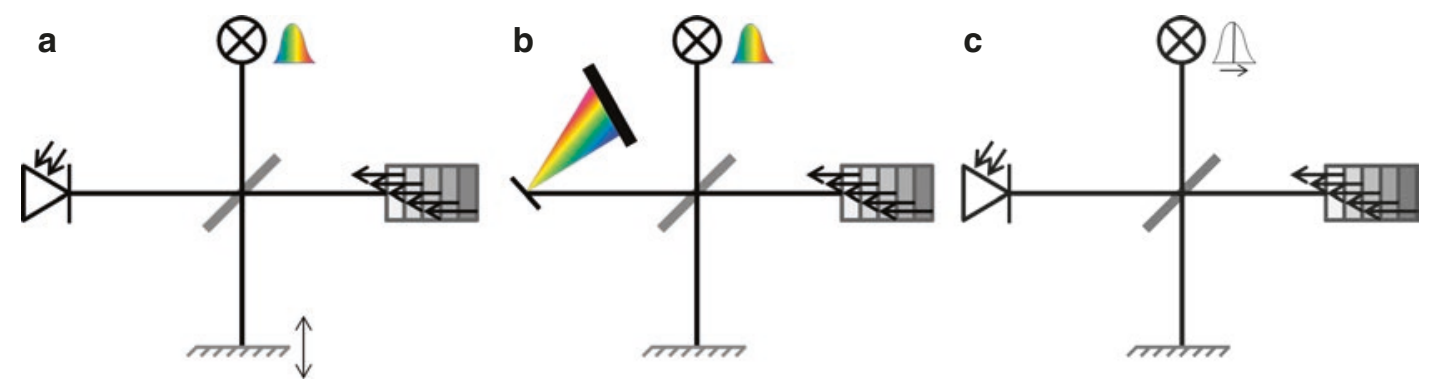

Fig. 14.5 Different types of OCT implementations. (a) Time-domain OCT with broadband light source and moveable mirror for depth ranging and a photodiode for detection. (b) Fourier-domain setup with broadband light source, fixed mirror and spatially encoded detection. (c) Swept-source OCT setup with tunable light source, fixed reference mirror and a photodiode for detection

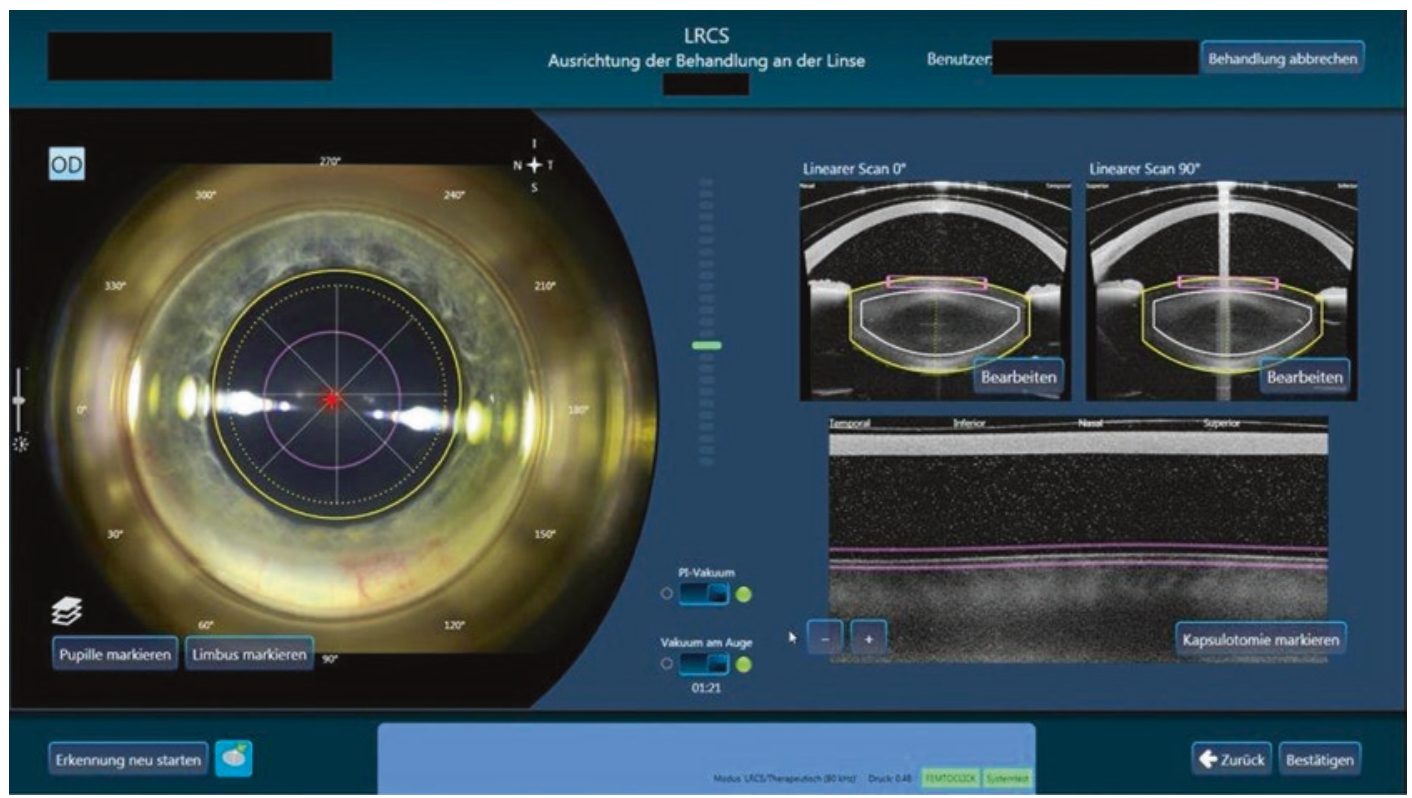

Fig. 14.6 Alignment Screen of the VICTUS laser system

whole A-scan can be obtained by simply taking the Fourier transform of the acquired spectra without any moving parts [15].

This allows for at least two orders of magnitude higher A-scan rates of several $100 \mathrm{KHz}$, while the signal to noise ratio (SNR) is improved proportional to the number of detectors (camera pixels of the line sensor, or number of samples with swept-source OCT, both typically around 2000). Therefore Fourier-domain OCT has both a speed and sensitivity advantage of about $20 \mathrm{~dB}$ over time-domain OCT [16].
While retinal imaging of the human eye is the most common clinical application of OCT, anterior segment imaging becomes increasingly popular. Obviously the wavelength of the light source needs to be optimized for the application. For anterior segment applications, the $1300 \mathrm{~nm}$ range is favorable because of its deeper penetration of the sclera, which allows for better chamber angle visualization. Anterior segment OCT has great potential for refractive surgery applications and especially for cataract surgery (Fig. 14.6). 


\subsection{Treatment Steps of FLACS Procedure}

\subsubsection{Planning}

A series of parameters, such as pupil dilation, lens thickness, and corneal thickness, will need to be measured before a cataract surgery. Then a surgical plan will be created. Normally, the planning parameters include the size, shape, and desired center of laser ablation for capsulotomy. Further planning parameters are diameter, depth, and patterns of cut for lens fragmentation. The planning will include also location, depth, and architecture of corneal incisions. Adjustments still can be performed in real-time guiding by cross-sectional imaging during cataract surgery. Consider the LenSx laser system. After the system warm up and self-checks have been completed, the opening screen will appear on the monitor (Fig. 14.7). The surgeon then presses program button to go to the patternselection screen as shown in Fig. 14.7. There, the surgeon will program the lens pattern, capsulotomy pattern, primary incision pattern, secondary incision pattern and arcuate incision pattern. Each of these patterns may be used either individually or consecutively during the same procedure.

\subsubsection{Engagement}

The patient interface optically couples the eye to the laser delivery system to prevent eye movement. This is the first clinical step for FLACS. The docking system, namely the patient interface, is normally composed of a curved applanation lens and suction ring. Both parts are integrated into a single piece and mounted on the laser delivery system. The patient interface serves as a sterile barrier between the patient's eye and the femtosecond laser. An ideal patient interface should satisfy three requirements. First, it should fix the eye without distorting it and causing the intraocular pressure (IOP) to increase. Second, it should have a wide field of view to allow for surgical facility. Third, it should have the ability to prevent corneal folds that occur with suction, and allow for a tight laser focus. All commercially available femtosecond lasers appear to be effective in stabilizing the patient' eye; however, the methods and devices for docking are an area of differentiation for these femtosecond lasers [17]. LenSx and Victus have reported a curved applanation lens and suction system. Catalys and LensAR have reported a fluid filled suction

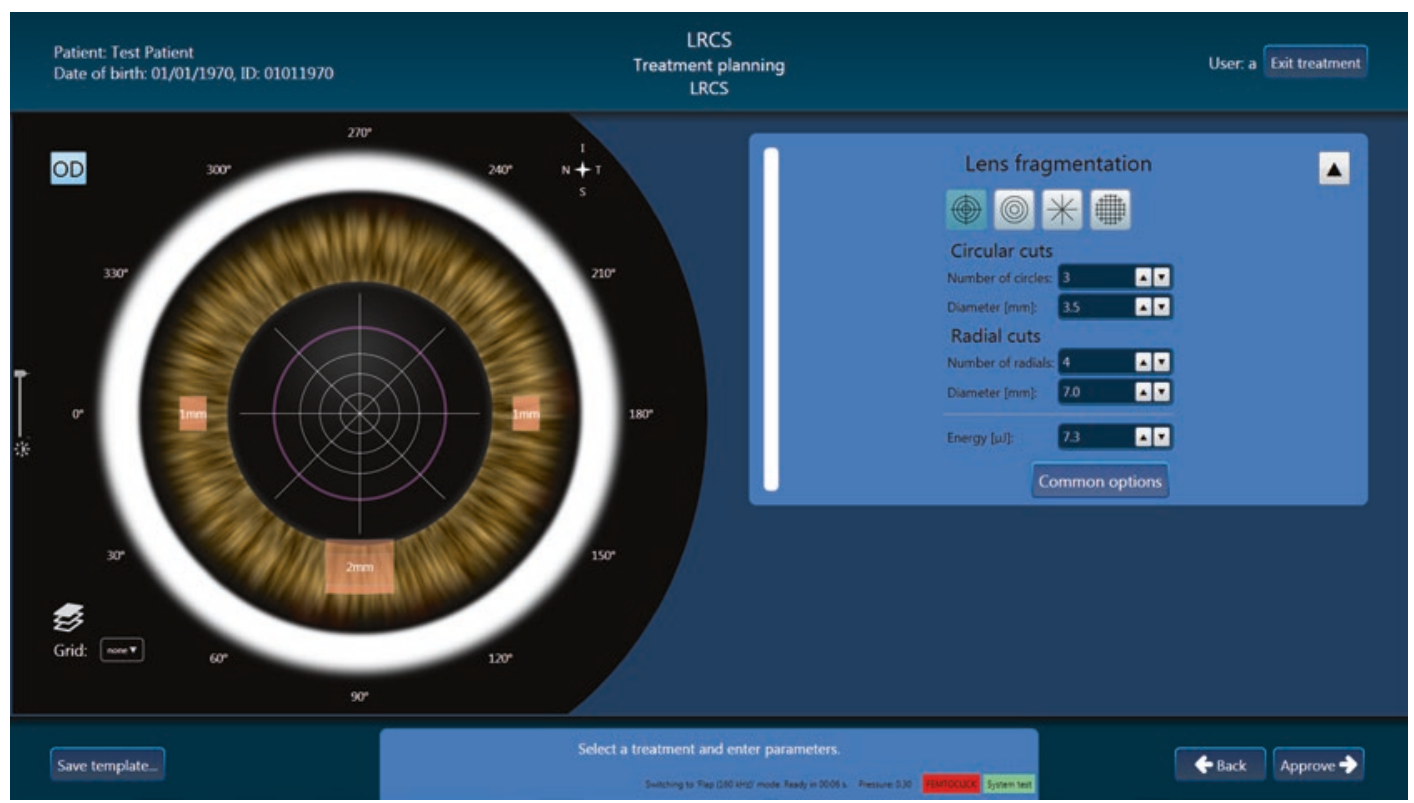

Fig. 14.7 Treatment planning screen of the VICTUS laser system 
ring. LensAR has reported a water bath suction fixation device [18]. Femto LDV has reported a liquid interface [19].

\subsubsection{Visualization and Customization}

The image guidance sub-system gives the instruction about the dimensions and location of ocular structures. It is a critical part of femtosecond laser cataract surgery because it guides the surgeon through lens fragmentation zones and the placement of incisions. This sub-system must be able to detect the iris boundaries so that the surgery can be made safely without cutting the iris. To maintain a safety zone and not cut in the posterior capsule, this sub-system must be able to detect the posterior surface of the lens. The corneal thickness should be measured in order to customize corneal incisions for each patient. The noncontact, fast method of high-resolution image acquisition with FD-OCT, which enables detailed cross-sectional imaging of the anterior segment, is useful in various clinical settings. Some important parameters for cataracts, such as iris boundaries, corneal thickness, lens position, and iridocorneal angle, can be measured by FD-OCT in real time [20, 21]. The advantages of FD-OCT, including noncontact, high resolution, accuracy in the presence of corneal opacity, and ease of use, make it the most popular sub-system for commercially available femtosecond lasers in cataract surgery. The live OCT images are used to help the doctor in the docking process. The surgeon also uses these OCT images to adjust and verify the position and orientation of the selected surgical patterns. The platforms that use FD-OCT for three-dimensional, high-resolution viewing of ocular structures in cataract surgery include LenSx, Catalys, Victus, and FemtoLDV, whereas LensAR utilizes Scheimpflug imaging technology.

\subsubsection{Treatment}

Treatment is the final step of femtosecond-laser cataract surgery. The femtosecond laser creates incisions through tightly focused pulses that cut eye tissue with micron-scale precision. The incision is achieved by contiguously placing the femtosecond laser focus scanned by a computercontrolled delivery system through the laser spot pattern from posterior to anterior, which will reduce the amount of radiation reaching the retina. The femtosecond laser beam creates individual photodisruption sites in a contiguous pattern to form continuous incisions. The spacing between each spot is programmed by the surgeon, who enters the size, shape, and location of the scanning pattern before the treatment. The treatment includes four parts, namely, clear corneal incisions, creation of the capsulotomy, fragmentation of the lens nucleus, and correction of astigmatism through corresponding arcuate incisions. A corneal incision consists of a series of cuts starting at the desired corneal thickness and continuing through the surface of the cornea. A capsulotomy incision consists of a cylindrical cut starting from below the surface of the anterior capsule and continuing through the capsule a few microns into the anterior chamber. A lens fragmentation incision consists of a few oriented elliptical shaped planes that intersect at the center of the lens and the maximum cutting depth should be above the posterior capsule. The surgeon controls the femtosecond laser primarily by a console interface comprised of a keyboard, touchpad, and monitor. Pattern parameters are entered at the console interface by the surgeon. A footswitch allows the surgeon to start and stop femtosecond laser treatment. The surgeon can interrupt the procedure at any time by releasing the footswitch and resume it by repressing the footswitch. The femtosecond laser will continue the treatment until the programmed pattern is complete. For the LenSx system, the lens fragmentation pattern performs phacofragmentation of the crystalline lens and may be specified as either chopped or cylindrical patterns, or both. The first pattern is usually the choice for harder lenses and the second pattern for softer ones. A chopped pattern creates intersecting radial lines at the programmed posterior depth inside the lens. When one layer is filled, the laser creates another layer a few microns above to fill in the 
ellipsoidal shape, by creating vertical, ellipsoidal planes that intersect at right angles. When the cylindrical pattern is used, the treatment pattern starts at the programmed posterior depth and continues to the programmed anterior depth as a series of concentric rings. When the programmed anterior depth is reached, the pattern will be completed. The patterns can also be combined to form a hybrid pattern. In this case, both the intersecting radial lines for the chopped version and a series of concentric rings for the cylindrical version will be created at the programmed posterior depth. Both patterns are burned simultaneously for each layer until the anterior depth is reached as programmed.

After the application of a femtosecond laser, a standard manual PE or Phaco will be employed to remove the broken crystalline lens. After the removal of the cataract, an IOL is usually implanted into the eye. The cataract surgery is then performed, and the patient's cloudy natural lens is removed and replaced with a synthetic lens to restore vision.

\subsubsection{Benefits}

The first clinical report of a human eye treated by femtosecond laser cataract surgery was in Hungary in 2008 [12]. It is a promising new technological advance, which plays an ever increasing role in cataract surgery. The FDA approved the usage of femtosecond laser for cataract surgery in 2010. The application of a femtosecond laser in cataract surgery increased dramatically after the LenSx system was cleared for use. Just in a few years, femtosecond lasers have become relevant in cataract surgery in clinic as an opportunity to improve the quality of the surgical procedure. In a relatively short period of time, the LenSx femtosecond laser system has been used in more than 200,000 procedures worldwide to date [13]. The accumulating clinical experience with FLACS indicates that it offers several potential advantages over manual surgery: a better quality of incision with reduced induced astigmatism, increased reliability and reproducibility of the capsulotomy with increased stability of the implanted lens, and a reduction in the use of ultrasound [22].

\subsubsection{Corneal Incision}

Clear corneal self-sealing incision architecture is of paramount importance [22]. Previous study shows the corneal incision with internal wound gape will increase the risk of leakage and thus postoperative endophthalmitis [23]. Manual corneal incisions are difficult to control in terms of length and architecture. Such manually created incisions may affect the stability of the wound under pressure following surgery and potentially allow leakage [24]. One of the most important advantages of FLACS is that the corneal incisions can be designed to construct reproducible and stable incisions, so that the incision width and length may be customized according to this precept with a high degree of integrity $[18,24]$. Favorable results of more stable and aberration-free results from FLACS were reported in the triplanar configuration [25]. One of the benefits from FLACS is that corneal wounds and arcuate incisions in the desired position and depth make the control of postoperative astigmatism much more effective [26].

\subsubsection{Capsulotomy}

If FLACS can produce a reproducibly round, centered, and intact anterior capsule, this alone would improve the safety of cataract surgery in a way that could possibly justify the introduction of this new technology [9]. The anterior capsular tears in the hands of experienced surgeons have a high incidence [27, 28]. Capsular rupture can lead to a rise in IOP, persistent uveitis, cystoid macular edema, retinal detachment, infection, and retained soft lens matter requiring removal [9]. Femtosecond lasers made it very easy to remove the capsular button [29]. A report based on a small group of porcine eyes showed that the capsule strength was as good as or greater than a manual capsulorhexis, enabling a greater force of stretch before rupture [12]. The importance of a precisely sized capsulorhexis to optimize IOL position and performance is well known. The major source of error in IOL power calculation is the effective lens position inaccuracy [30]. FLACS can produce a more precise, reproducible, better centered, and stronger opening of the anterior capsule than 
conventional manual continuous curvilinear capsulorhexis (CCC) [22, 31]. FLACS can create capsulotomy that is more precise, rounder, more regularly shaped, and with greater centration than a manual technique, which permits for a better IOL and capsule overlap. The improved overlap of the anterior capsule by the IOL has been shown to produce less IOL tilt and decentration compared with manual CCC [22]. Many similar results were obtained from commercially available femtosecond lasers in cataract surgery [18].

\subsubsection{Lens Fragmentation}

Femtosecond lasers are powerful tools for segmenting the crystalline lens, making the difficult chop steps that most frequently lead to complications in regular cataract surgery much easier [32, 33]. Femtosecond laser cuts on the crystalline lens soften the harder cataracts and reduce the amount of ultrasound energy from the PE probe, thereby diminishing the risk of capsule complications and corneal endothelial injury (Fig. 14.8). A report done on a porcine eye study with the LenSx platform stated that FLACS reduced PE power by $43 \%$ and operative time by $51 \%$ [12]. Such a comparison in human eye showed a 39\% average reduction in dispersed energy for PE [34]. A decrease in PE power and time using the LenSx platform was also reported by other studies. The effective $\mathrm{PE}$ time showed a $70 \%$ reduction in a study conducted on the Catalys platform [35]. A significant decrease in the PE power and time was reported by a comparative study conducted on the Victus platform [36]. To date, the clinical reports showed that the percentage reduction of FLACS PE varied by company and grade of cataract but was at least $33 \%$.

\subsubsection{Other Benefits}

Other benefits of FLACS include the ability to correct astigmatism through corresponding arcuate incisions, a reduction of infection possibility, decreased endothelial cell loss, and possibly improved visual and refractive outcomes.
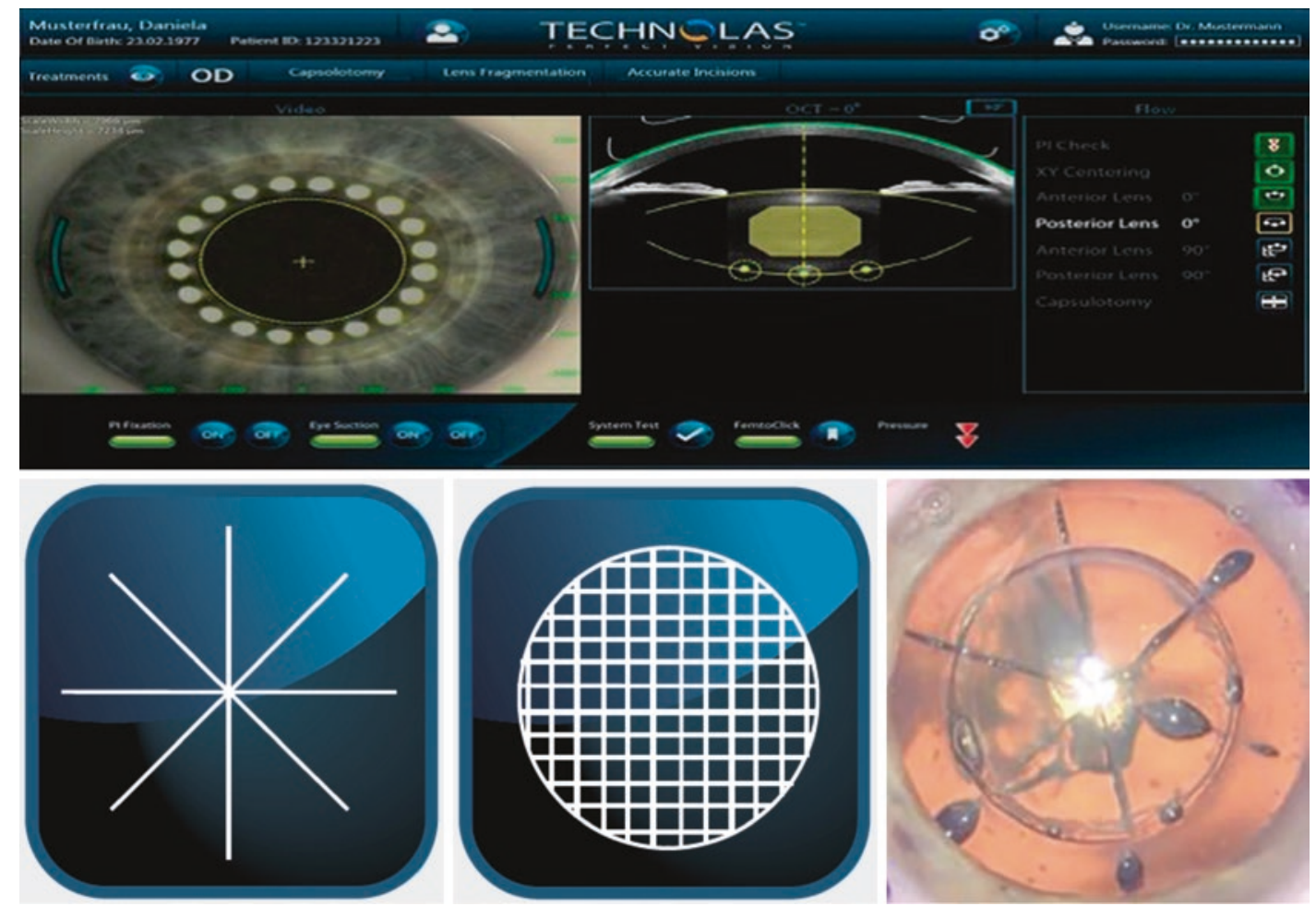

Fig. 14.8 Lens fragmentation pattern of the VICTUS femtosecond laser 


\subsubsection{Safety}

Multiple commercial femtosecond laser systems are approved by the FDA for refractive surgery, including use in creating corneal flaps in LASIK surgery and in cataract surgery. The laser safety is well evaluated for LASIK surgery through investigation from laboratory to clinic [3745]. The laser safety for FLACS also has been reported [46]. A safety assessment can provide an analysis of exposure relative to established exposure limits. ANSI Z136.1-2007 series provides internationally accepted exposure limits. According to ANSI Z136.1-2007, the photo disruption threshold for a femtosecond laser is about $1 \mathrm{~J} / \mathrm{cm}^{2}$. With a cataract procedure, for efficient cutting, the laser is tightly focused on the lens or cornea, which results in a transmitted beam with a diameter of approximately a few $\mathrm{mm}$ on the retina. Because the size of the beam on the retina is approximately a few $\mathrm{mm}$ in diameter (compared with the focused $2 \mu \mathrm{m}$ spot on the lens or cornea), the fluence on the retina is much smaller than in the lens or cornea and much lower than the photo disruption threshold. In fact, the actual temperature increase in an in vivo retina is expected to be even smaller due to the presence of heat sinks (vitreous, aqueous humor) and the cooling effect of local blood flow. Additionally, in case of cataract surgery, the laser energy reaching the retina may be even lesser due to the presence of the cataract, resulting in greater safety for the retina during FLACS. Experiments on rabbits did not show retinal damage with FLACS. Safety evaluation of femtosecond lentotomy on the porcine lens also showed that there is no retinal damage at the setting, which is very similar to FLACS [47]. Initial clinical results for safety in FLACS were reported in 2010 [46]. To date, more than 200,000 clinical experiences have shown the retina safety for FLACS in a persuasive way.

\subsection{Clinical Experience with FLACS}

The OCT is crucial to centration on the visual axis:

Femtosecond lasers for cataract surgery have been on the market for over 5 years now, but the questions for cataract surgeons are: Has the tech- nology advanced over this period, and if so, what advances have been made? With the latest $\mathrm{B}+\mathrm{L}$ VICTUS femtosecond laser platform (Fig. 14.9), a number of key features have been introduced that are considerably improving patient outcomes. The feature list is long, and cataract surgeons appreciate the VICTUS' ability to create LASIK flaps and perform different kinds of keratoplasty, but in terms of cataract surgery, there are a number of key features that are the most important ones in terms of improving patient outcomes.

The first key feature is the implementation of the new swept-source OCT system (Fig. 14.10). It has a very high resolution; it displays a live OCT image throughout the procedure and performs 50,000 A-scans per second. Surgeons view it as almost having a "filmic" quality. The system also has enhanced contrast sensitivity compared with previous instruments, and the new software offers the automatic recognition of the pupil, lens thickness and the anterior and posterior capsule. There's a long list of features: various software optimizations, not least an advanced identification management system; an improved OCT capability; soft docking, which is quite important for cataract surgery; and, perhaps most impressive of all, the new apex centration system, all of which can be seen in a surgical video, available at: https://youtu. be/-6VkDF0G7gQ (Author: Dr. Tobias Neuhann).

With all of the axes in the eye (Fig. 14.11), it can be hard to decide how to center laser capsulotomies or even more so, manual capsulorhexes. It's best to center the capsulotomy on the visual axis, and the OCT supports this by calculating $0^{\circ}$ and $90^{\circ}$ on the surfaces of the anterior and posterior capsules.

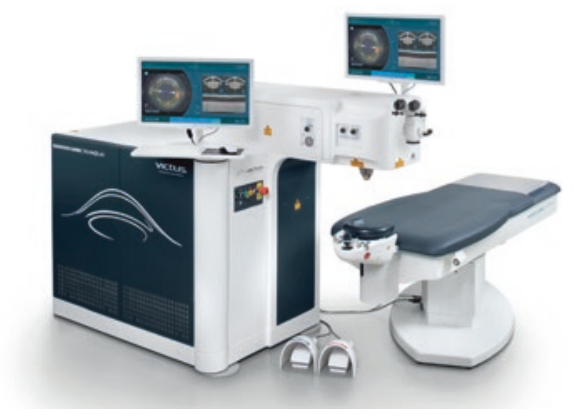

Fig. 14.9 VICTUS ${ }^{\circledast}$ Femtosecond Laser Platform 




Fig. 14.10 The new swept-source OCT system: 50,000 A-scans per second enhanced contrast sensitivity, plus automatic recognition of the pupil, lens thickness and the anterior and posterior capsule. See the OCT in action online as part of Femtosecond-Laser-Assisted cataract surgery at: youtube/-6VkDF0G7gQ
Temporal

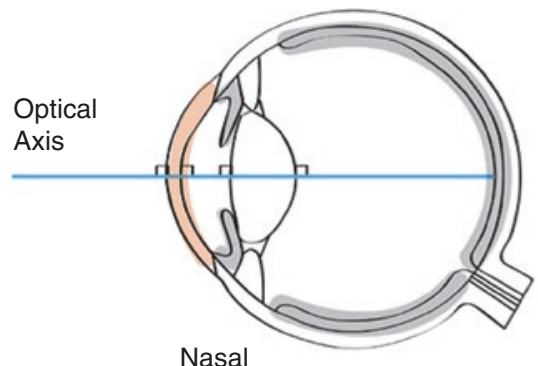

Temporal

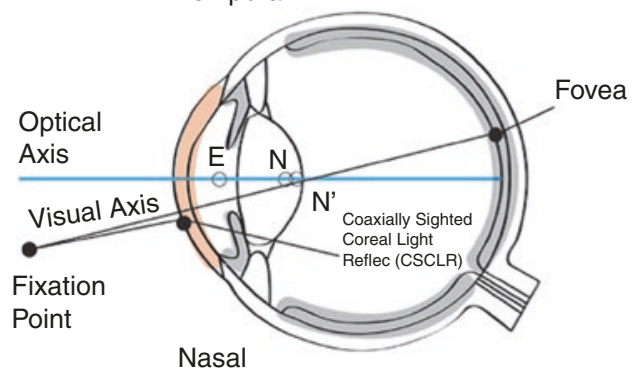

Fig. 14.11 The optical axis of the eye, as a purely theoretical construct where the surfaces of the cornea and crystalline lens are rotationally symmetric, and their centers of curvature lie on a common line. If a point source was shined into the eye, there would be a point where all the Purkinje images coincide-the line from the point source through each Purkinje image would define the optical axis. In real eyes, the Purkinje images do not align and the surfaces are not rotationally symmetric, so no true optical axis of the eye exists. Occasionally, the optical
Temporal



Temporal



axis is defined as the line that minimizes the deviation of the Purkinje images; (top right) The fovea, the center of the pupil, E, and the nodal points $\mathrm{N}$ and $\mathrm{N}^{\prime}$; (bottom left) coaxially sighted corneal light reflex (CSCLR), where the line from the fixation point that is normal to the cornea defines the CSCLR; (bottom right) The pupillary axis (perpendicular to the cornea, found by aligning the first Purkinje image with the center of the pupil) and the line of sight (connecting the fixation point to the center of the entrance pupil) 
Where the lines cross (Fig. 14.12), the surgeon centers the capsulotomy-which enables the surgeon to find the apex of the lens. Usually, most surgeons would center the capsulotomy on the pupil center, which is easiest - but there is a noticeable difference in positioning (Fig. 14.13).

Cataract surgeons are convinced, that using the VICTUS' OCT-guided method assures that the center of the lens is optimally positioned in the capsular bag, centered on the visual axis. This is particularly important for aspheric, toric and multifocal lenses, which is greatly improved, using the VICTUS apex centration capability. The optics of IOLs is becoming increasingly sophisticated, and apex centration should now be considered a mandatory tool for sophisticated IOLs such as aspheric monofocal, multifocal

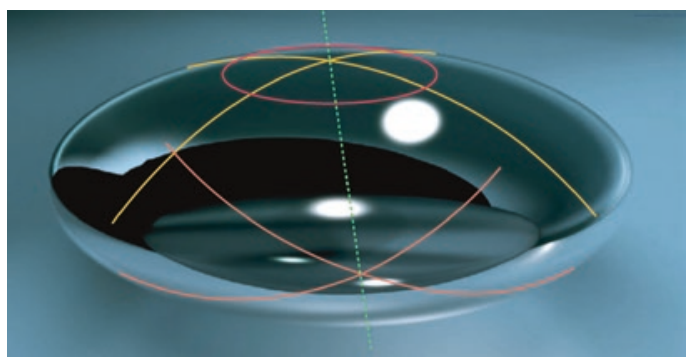

Fig. 14.12 VICTUS' OCT enables the cataract surgeon to center the capsulotomy on the visual axis by calculating $0^{\circ}$ and $90^{\circ}$ on the surface of the anterior and posterior capsules. The capsulotomy is centered where the lines cross, enabling the surgeon to find the apex of the lens

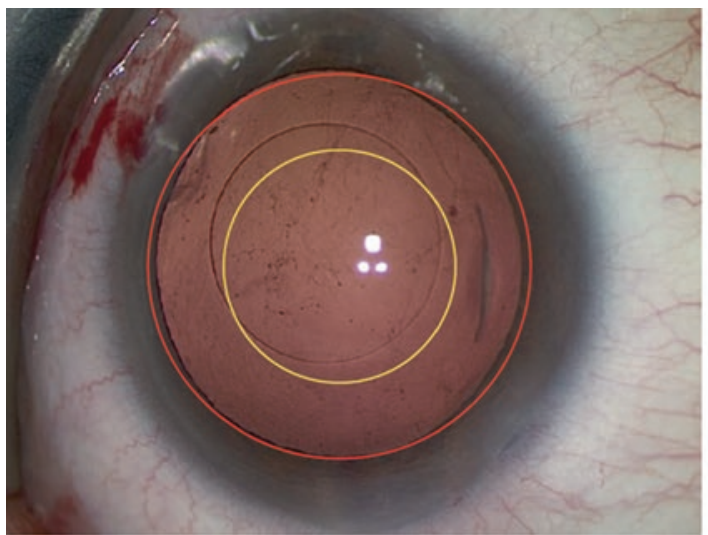

Fig. 14.13 Left: The difference between a capsulotomy centered on the pupil (red and yellow circles) and the apex of the lens (actual capsulotomy) as determined by OCT. Right: The same eye once the IOL is implanted. The or trifocal toric IOLs. The latest VICTUS system's high-resolution OCT is definitely superior to a Purkinje image and will be the 'conditio sine qua non' for the next generation of IOLs. It will enable more patients to benefit from the advantages of these IOLs, should help avoid the specter of negative dysphotopsia, especially the outer dark arc which patients often complain about and for which there is no real solution, and events like capsular phimosis and postoperative toric IOL rotation. Considering some of the most recent IOLs to come to the market with a groove in the optic edge, that "hooks" the lens in place at the anterior capsule; the benefits of the femtosecond laser rhexis approach become obvious: When this lens is implanted in a standard eye, via an apex-centered capsulotomy, phimosis can't occur, because the anterior capsule sits inside the lens, and the lens cannot rotate. Fixating the IOL on the anterior capsule means it is closer to the iris and hence will not create any negative dysphotopsia. Cataract surgeons believe that this procedure will further the next generation of IOL optics.

\subsection{Summary and Outlook}

The advent of all-solid-state femtosecond lasers, coupled with a computer-controlled beam delivery system, enables the new applications of high

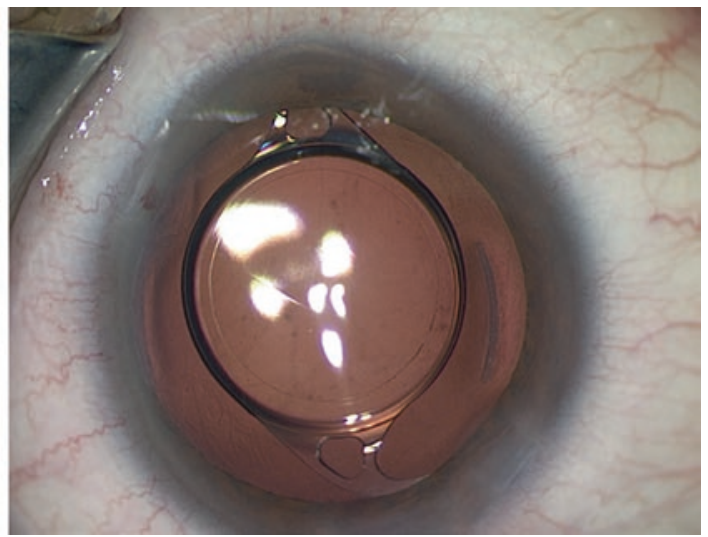

IOL looks decentered-but is not. The symmetry between the edge of the anterior capsule and the edge of the implant proves that an apex-centered capsulotomy is superior to a pupil-centered capsulotomy 
precision femtosecond laser ablation for ophthalmology. Combining this with a precision imaging technique for anterior segment, such as an OCT system, can make it possible to accurately target tissue in a crystalline lens by a femtosecond laser. Femtosecond laser systems have successfully entered the cataract surgery market as a promising new technological advance, which plays an ever increasing role in cataract surgery, where it automates the three main surgical steps: corneal incision, capsulotomy, and lens fragmentation. In just in a few years, femtosecond lasers have become relevant in cataract surgery in clinic as an opportunity to improve the quality of the surgical procedure. In a relatively short period of time, FLACS has been used in more than 200,000 procedures worldwide to date [13]. Clinical experiences so far indicate that this new technology is promising for the field of cataract surgery. FLACS appears safe and efficacious, and may eventually be proven superior to conventional cataract surgery.

\section{References}

1. Chylack LT, Leske C, Sperduto R, et al. Lens opacities classification system. Arch Ophthalmol. 1988; https:// doi.org/10.1001/archopht.1988.01060130356020.

2. Sparrow JM, Bron AJ, Brown NA, et al. The Oxford clinical cataract classification and grading system. Int Ophthalmol. 1986; https://doi.org/10.1007/ BF00137534.

3. West SK, Rosenthal F, Newland HS, Taylor HR. Use of photographic techniques to grade nuclear cataracts. Investig Ophthalmol Vis Sci. 1988;29:73-7.

4. Bille JF, Schanzlin D. Method for removing cataractous material. US Patent. 1993;5, 246, 435.

5. Loesel FH, Tien A-C, Backus SJ, et al. Effect of reduction of laser pulse width from 100 ps to 20 fs on the plasma-mediated ablation of hard and soft tissue. In: Thermal therapy, laser welding, and tissue interaction; 1999. https://doi.org/10.1117/12.335791.

6. Cook CE. Pulse compression-key to more efficient radar transmission. Proc IRE. 1960; https://doi. org/10.1109/JRPROC.1960.287599.

7. Strickland D, Mourou G. Compression of amplified chirped optical pulses. Opt Commun. 1985; https:// doi.org/10.1016/0030-4018(85)90120-8.

8. Han M, Giese G, Zickler L, et al. Mini-invasive corneal surgery and imaging with femtosecond lasers. Opt Express. 2004; https://doi.org/10.1364/ OPEX.12.004275.
9. Hodge C, Bali SJ, Lawless M, et al. Femtosecond cataract surgery: a review of current literature and the experience from an initial installation. Saudi J Ophthalmol. 2012;

10. Leaming DV. Practice styles and preferences of ASCRS members-2002 survey. J Cataract Refract Surg. 2003;29:1412-20.

11. Palanker DV, Blumenkranz MS, Andersen D, et al. Femtosecond laser-assisted cataract surgery with integrated optical coherence tomography. Sci Transl Med. 2010; https://doi.org/10.1126/scitranslmed.3001305.

12. Nagy Z, Takacs A, Filkorn T, Sarayba M. Initial clinical evaluation of an intraocular femtosecond laser in cataract surgery. J Refract Surg. 2009; https://doi.org/ 10.3928/1081597X-20091117-04.

13. Nagy ZZ, Mastropasqua L, Knorz MC. The use of femtosecond lasers in cataract surgery: review of the published results with the LenSx system. J Refract Surg. 2014; https://doi.org/10.3928/10815 97X-20141021-04.

14. Huang D, Swanson EA, Lin CP, et al. Optical coherence tomography. Science. 1991; https://doi. org/10.1002/jcp.24872.The.

15. Fercher AF, Hitzenberger CK, Kamp G, El-Zaiat SY. Measurement of intraocular distances by backscattering spectral interferometry. Opt Commun. 1995; https://doi.org/10.1016/0030-4018(95)00119-S.

16. Choma M, Sarunic M, Yang C, Izatt J. Sensitivity advantage of swept source and Fourier domain optical coherence tomography. Opt Express. 2003; https:// doi.org/10.1364/OE.11.002183.

17. He L, Sheehy K, Culbertson W. Femtosecond laserassisted cataract surgery. Curr Opin Ophthalmol. 2010; https://doi.org/10.1097/ICU.0b013e3283414f76.

18. Alió JL, Abdou AA, Puente AA, et al. Femtosecond laser cataract surgery: updates on technologies and outcomes. J Refract Surg. 2014; https://doi.org/10.3 928/1081597X-20140516-01.

19. Pajic B, Vastardis I, Gatzioufas Z, Pajic-Eggspuehler B. First experience with the new high-frequency femtosecond laser system (LDV Z8) for cataract surgery. Clin Ophthalmol. 2014; https://doi.org/10.2147/ OPTH.S72983.

20. Doors M, Berendschot TTJM, de Brabander J, et al. Value of optical coherence tomography for anterior segment surgery. J Cataract Refract Surg. 2010; https://doi.org/10.1016/j.jcrs.2010.05.002.

21. Kim HY, Budenz DL, Lee PS, et al. Comparison of central corneal thickness using anterior segment optical coherence tomography vs ultrasound pachymetry. Am J Ophthalmol. 2008;145:228-232.e1. https://doi. org/10.1016/j.ajo.2007.09.030.

22. Abouzeid H, Ferrini W. Femtosecond-laser assisted cataract surgery: a review. Acta Ophthalmol. 2014; https://doi.org/10.1111/aos.12416.

23. Chee SP, Ti SE, Lim L, et al. Anterior segment optical coherence tomography evaluation of the integrity of clear corneal incisions: a comparison between 2.2$\mathrm{mm}$ and 2.65-mm main incisions. Am J Ophthalmol. 2010; https://doi.org/10.1016/j.ajo.2009.12.008. 
24. Masket S, Sarayba M, Ignacio T, Fram N. Femtosecond laser-assisted cataract incisions: architectural stability and reproducibility. J Cataract Refract Surg. 2010; https://doi.org/10.1016/j.jcrs.2010.03.027.

25. Alió JL, Abdou AA, Soria F, et al. Femtosecond laser cataract incision morphology and corneal higher-order aberration analysis. J Refract Surg. 2013; https://doi. org/10.3928/1081597X-20130819-01.

26. Nagy ZZ, Takacs AI, Filkorn T, et al. Complications of femtosecond laser-assisted cataract surgery. J Cataract Refract Surg. 2014; https://doi.org/10.1016/j. jcrs.2013.08.046.

27. Marques FF, Marques DMV, Osher RH, Osher JM. Fate of anterior capsule tears during cataract surgery. J Cataract Refract Surg. 2006; https://doi. org/10.1016/j.jcrs.2006.05.013.

28. Ng DT, Rowe NA, Francis IC, et al. Intraoperative complications of 1000 phacoemulsification procedures: a prospective study. J Cataract Refract Surg. 1998; https://doi.org/10.1016/S0886-3350(98)80235-6.

29. Tackman RN, Villar Kuri J, Nichamin LD, Edwards K. Anterior capsulotomy with an ultrashort-pulse laser. J Cataract Refract Surg. 2011; https://doi. org/10.1016/j.jcrs.2010.11.030.

30. Norrby S. Sources of error in intraocular lens power calculation. J Cataract Refract Surg. 2008; https://doi. org/10.1016/j.jcrs.2007.10.031.

31. Friedman NJ, Palanker DV, Schuele G, et al. Femtosecond laser capsulotomy. J Cataract Refract Surg. 2011; https://doi.org/10.1016/j. jcrs.2011.04.022.

32. Artzén D, Lundström M, Behndig A, et al. Capsule complication during cataract surgery: case-control study of preoperative and intraoperative risk factors. Swedish Capsule Rupture Study Group report 2. J Cataract Refract Surg. 2009; https://doi.org/10.1016/j. jers.2009.05.026.

33. Gauba V, Tsangaris P, Tossounis C, et al. Human reliability analysis of cataract surgery. Arch Ophthalmol. 2008;126:173. https://doi.org/10.1001/ archophthalmol.2007.47.

34. Talamo JH, Gooding P, Angeley D, et al. Optical patient interface in femtosecond laser-assisted cataract surgery: contact corneal applanation versus liquid immersion. J Cataract Refract Surg. 2013; https://doi. org/10.1016/j.jcrs.2013.01.021.

35. Abell RG, Kerr NM, Vote BJ. Femtosecond laserassisted cataract surgery compared with conventional cataract surgery. Clin Exp Ophthalmol. 2013; https:// doi.org/10.1111/ceo.12025.

36. Reddy KP, Kandulla J, Auffarth GU. Effectiveness and safety of femtosecond laser-assisted lens fragmentation and anterior capsulotomy versus the manual technique in cataract surgery. J Cataract Refract Surg. 2013; https://doi.org/10.1016/j.jcrs.2013.05.035.

37. Juhasz T, Nagy Z, Sarayba M, Kurtz RM. Femtosecond lasers in ophthalmology: clinical applications in anterior segment surgery. Proc SPIE. 2010:758904758909. https://doi.org/10.1117/12.847895.

38. Schumacher S, Sander M, Stolte A, et al. Investigation of possible fs-LASIK induced retinal damage. Proc SPIE. 2006; https://doi.org/10.1117/12.645147.

39. Sun H, Fan Z, Yan Y, et al. Femtosecond laser subsurface scleral treatment in cadaver human sclera and evaluation using two-photon and confocal microscopy. Proc SPIE. 2016; https://doi. org/10.1117/12.2197912.

40. Sun H, Hosszufalusi N, Juhasz T, Mikula ER. Simulation of the temperature increase in human cadaver retina during direct illumination by $150-\mathrm{kHz}$ femtosecond laser pulses. J Biomed Opt. 2011;16:108001-9.

41. Sun H, Kurtz RM, Juhasz T. Simulation the temperature increase in porcine cadaver iris during direct illumination by femtosecond laser pulses. J Cataract Refract Surg. 2012; https://doi.org/10.1016/j. jers.2010.09.018.

42. Sun H, Kurtz RM, Juhasz T. Measurement of the temperature increase in the porcine cadaver iris during direct illumination by femtosecond laser pulses. J Biomed Opt. 2010:756216-756217. https://doi. org/10.1117/1.JBO.17.7.078001.

43. Sun H, Kurtz RM, Juhasz T. Finite element model of the temperature increase in excised porcine cadaver iris during direct illumination by femtosecond laser pulses. J Biomed Opt. 2012;17:78001-6.

44. Sun H, Kurtz RM, Mikula ER, Juhasz T. Temperature increase in porcine cadaver iris during direct illumination by femtosecond laser pulses. J Cataract Refract Surg. 2011; https://doi.org/10.1016/j. jcrs.2010.09.018.

45. Sun H, Mikula E, Kurtz RM, Juhasz T. Temperature increase in human cadaver retina during direct illumination by femtosecond laser pulses. J Refract Surg. 2010;26:272-7. https://doi.org/10.3928/10815 97X-20090617-08.

46. Zhang J, Wang R, Chen B, et al. Safety evaluation of femtosecond lentotomy on the porcine lens by optical measurement with 50 -femtosecond laser pulses. Lasers Surg Med. 2013; https://doi.org/10.1002/ lsm.22154.

47. Sun H. Femtosecond-laser-assisted LASIK eye surgery and imaging. Proc SPIE. 2015; https://doi. org/10.1117/3.2205866. 
Open Access This chapter is licensed under the terms of the Creative Commons Attribution 4.0 International License (http://creativecommons.org/licenses/by/4.0/), which permits use, sharing, adaptation, distribution and reproduction in any medium or format, as long as you give appropriate credit to the original author(s) and the source, provide a link to the Creative Commons license and indicate if changes were made.

The images or other third party material in this chapter are included in the chapter's Creative Commons license, unless indicated otherwise in a credit line to the material. If material is not included in the chapter's Creative Commons license and your intended use is not permitted by statutory regulation or exceeds the permitted use, you will need to obtain permission directly from the copyright holder.

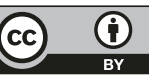

